In der Rubrik „Literatur kompakt" werden die wichtigsten Originalarbeiten aus der internationalen Fachliteratur referiert.

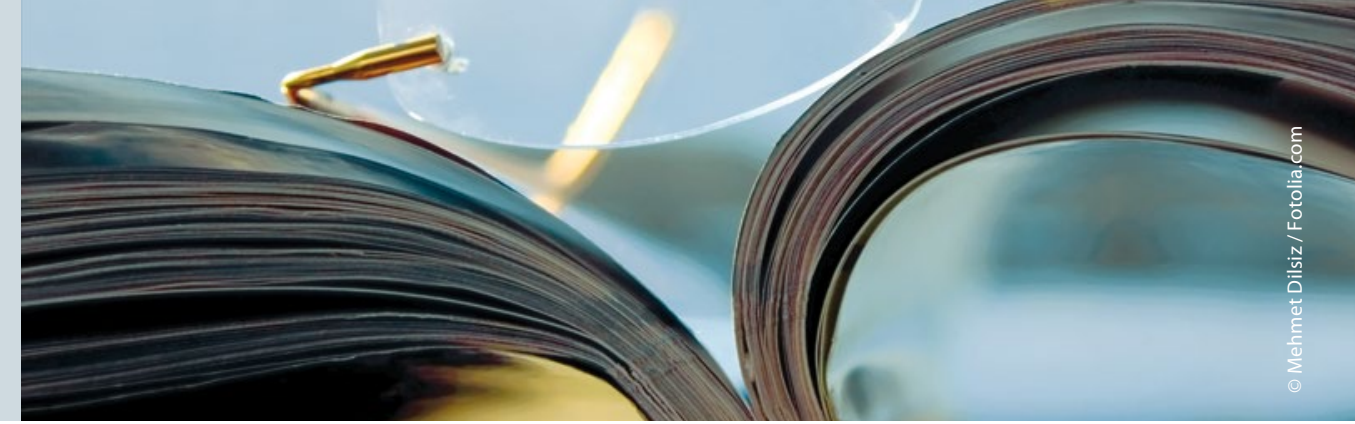

\section{Osteoporose-bedingte Wirbelfraktur: Frühe Vertebroplastie lindert den Schmerz}

\author{
Wirbelfrakturen sind eine häufige Komplikation der Osteoporose bei älteren \\ Menschen, insbesondere nach Stürzen. Ein Teil dieser Patienten entwickelt \\ chronische Schmerzen, die auch anhalten, wenn die Fraktur verheilt ist. \\ Australische Ärzte haben untersucht, inwieweit die Vertebroplastie bei akuten \\ schmerzhaften osteoporotischen Wirbelfrakturen analgetisch wirkt.
}

n der Vergangenheit hatten zwei randomisierte Studien in den USA keinen Nutzen der Vertebroplastie gefunden, wenn diese in einem Zeitraum von zwölf Monaten nach dem initialen Ereignis durchgeführt wurde. Das australische Ärzteteam um W. Clark vermutete, dass die negativen Studienergebnisse darauf zurückzuführen waren, dass bei der Mehrzahl der behandelten Patienten in diesen Studien die Fraktur bereits soweit konsolidiert war, dass keine schmerzlindernde Wirkung der Vertebroplastie mit tels Injektion von Polymethylmethacrylat mehr eintrat. Sie entschlossen sich daher, eine randomisierte Studie durchzuführen, in der die Patienten innerhalb von sechs Wochen nach der osteoporotischen Wirbelkörperfraktur eine Vertebroplastie erhielten.

Die multizentrische, randomisierte doppelblinde und placebokontrollierte Studie wurde an vier Krankenhäusern in Sydney, Australien, durchgeführt. Eingeschlossen wurden Patienten mit ein oder zwei osteoporotischen Wirbelkörperfrakturen, die weniger als sechs Wochen zurücklagen. Die Intensität der Rückenschmerzen musste auf einer Skala von 0-10 zwischen 7-10 liegen. Die Patienten wurden in Sedierung behandelt. In der Placebogruppe erfolgte eine
Scheintherapie. Der primäre Endpunkt der Studie war der Prozentsatz der Patienten, bei denen die Rückenschmerzen 14 Tage nach dem Eingriff 4 oder weniger auf einer Skala von 0-10 betrugen.

Ergebnisse: Im Zeitraum zwischen November 2011 und Dezember 2014 wurden 120 Patienten eingeschlossen. Die Studienteilnehmer waren im Mittel 80 Jahre alt und zu über $70 \%$ Frauen. Zwei Drittel der Patienten hatten bereits in der Vergangenheit osteoporotische Wirbelfrakturen erlitten. Die mittlere Dauer zwischen Fraktur und Behandlung betrug 2,5 Wochen. Die meisten Frakturen waren im thorakolumbalen Übergangsbereich aufgetreten. Die mittlere Schmerzintensität wurde auf einer Skala von 0-10 mit 8,6 angegeben. $24 \mathrm{der}$ 61 Patienten in der Vertebroplastiegruppe $(44 \%)$ hatten eine signifikante Schmerzbesserung nach zwei Wochen, in der Placebogruppe 12 von 59 (21\%). Dieser Unterschied war statistisch signifikant. In jeder Gruppe verstarben drei Patienten an Erkrankungen, die unabhängig von der Behandlung waren. Der Therapieeffekt hielt über sechs Monate Patienten waren allerdings nicht signifikant beeinflusst, ebenso wenig die Einnahme von Analgetika. an. Die Lebensqualitätsparameter der
Fazit: Bei Patienten mit osteoporotischen Wirbelfrakturen ist eine früh durchgeführte Vertebroplastie zur Schmerzreduktion wirksamer als eine Placebointervention.

Clark W, Bird P, Gonski P et al. Safety and efficacy of vertebroplasty for acute painful osteoporotic fractures (VAPOUR): a multicentre, randomised, double-blind, placebo-controlled trial. Lancet. 2016; 388: 1408-16

\section{Kommentar}

Die Vertebroplastie zur Behandlung von Schmerzen nach osteoporotischen Frakturen war nach den negativen Ergebnissen der beiden randomisierten Studien im Jahr 2009 fast vollständig aus der Schmerztherapie verschwunden. In der aktuellen Studie wurden die Patienten sehr viel früher als in den beiden vorhergehenden Studien behandelt. Der Therapieeffekt war signifikant und hielt bis zu sechs Monate an. Besonders erwähnenswert ist, dass es sich hier überwiegend um sehr alte Patienten mit hoher Schmerzintensität handelte. Bei dieser Population ist es besonders wichtig, durch eine Schmerzreduktion eine möglichst rasche Mobilisierung zu erreichen. Es bleibt abzuwarten, ob die Vertebroplastie wieder Eingang in die Schmerztherapie in Deutschland findet.

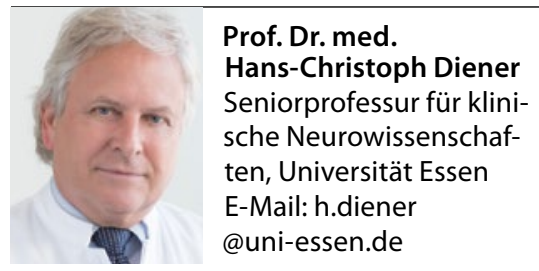

